\title{
Clinical course of fascial fibromatosis, vascularization and tissue composition of Palmar Aponeurosis in patients with Dupuytren's Contracture and concomitant arterial hypertension
}

\author{
Nathalia Shchudlo" ${ }^{1}$ Tatyana Varsegova ${ }^{2}$, Tatyana Stupina ${ }^{2}$, Michael Shchudlo ${ }^{1 *}$, Nathalia Shihaleva ${ }^{1}$ and Vadim Kostin ${ }^{1}$ \\ ${ }^{1}$ Clinics and experimental laboratory for reconstructive microsurgery and hand surgery \\ ${ }^{2}$ Laboratory of morphology, of FSBI (Federal State Budget Institution) Russian Ilizarov Scientific Center "Restorative Traumatology and Orthopaedics", Kurgan, \\ 640014, Russia
}

\begin{abstract}
Objective: Analysis of clinical course of palmar fascial fibromatosis (PFF) and histomorphometric characteristics of palmar aponeurosis in patients with Dupuytren's contracture (DC) with normal blood pressure (DCN) or with concomitant arterial hypertension (DCH).

Materials and methods: Case reports and histologic operation material from 140 Dupuytren's contracture patients treated in FSBI (Federal State Budget Institution) Russian Ilizarov Scientific Center "Restorative Traumatology and Orthopaedics" in 2014-2018. Inclusion criteria - men aged 43-77 years. Control - fragments of palmar aponeurosis from patients with acute open hand trauma.

Results: In DCH group PFF duration was insignificantly bigger ( $p>0,05)$, patients were older by 5 years at the beginning of PFF and by 7 years at the time of surgery, respectively $(\mathrm{p}<0,001)$ - compared to DCN group. Stage of contracture was $3(2 \div 3)$ in DCH and $2,5(2 \div 3)$ in DCN (p<0,05). In comparison with control in arteries of palmar aponeurosis pf DC patients the external diameter and lumen diameter were decreased but intima thickness increased. In comparison with DCN in DCH group luminal diameter was increased but intima thickness decreased $(p<0,05)$. Aponeurosis vascularity in DCH was less than in DCN ( $<<0,001)$ but per cents of vessels with inflammatory cells infiltration and fibrosis were increased ( $p<0,001)$, content of dense connective tissue was greater ( $p<0,001)$, but less hyperplastic connective tissue $(\mathrm{p}<0,001)$.

Conclusions: 1 . Patients with Dupuytren's contracture and concomitant pharmacologically controlled arterial hypertension are characterized by a later onset of the palmar fascial fibromatosis than at normal arterial pressure. This is presumably associated with the less severe constrictive remodeling of the perforating arteries of the palmar aponeurosis identified in this study, which was delayed by antihypertensive vasodilating therapy. 2 . In cases with concomitant arterial hypertension clinical and pathological data indicate accelerated development of fascial fibromatosis, which is caused by the rarefication of the microvasculature.
\end{abstract}

\section{Introduction}

Dupuytren's contracture belongs to fascial fibromatoses. Clinical symptoms begin with skin and palmar aponeurosis thickening as a result of the formation of fibroblast and myofibroblast proliferation focuses with their subsequent transformation into cords that limit the extension of the fingers. Progressive fingers contracture leads to osteoarthrosis of hand joints. This nosological form is socially significant because it reduces the life quality and professional skills of people in working age [1]. The disease has a polyetiological nature: the roles of genetic predisposition, microtraumas in manual labor, as well as the aggravating importance of extreme smoking and alcohol abuse were proved [2]. Its maximal prevalence was marked among the men of the Nordic Europe countries, prevailing age of patients from 40 to 70 years [3]. For the correction of the initial stages of Dupuytren's contracture, minimally invasive injection treatment techniques and percutaneous fasciotomy are used, but with advanced stages of hand deformity, removal of pathologically changed tissues by open fasciectomy is indicated [4]. With any method of treatment there is a likelihood of relapse, which is problematic to prevent because the effective methods of pathogenetic additive treatment are not developed. The main factors in the pathogenesis of fibromatosis are vasoconstriction, tissue hypoxia, free radical oxidation [5] and autoimmune inflammation [6]. Along with the narrowing of the vessels of the microvasculature the constrictive remodelling of palmar aponeurotic arteries and hemodinamic signs of vasoconstriction of superficial arterial palmar arc were revealed [7].

Dupuytren's contracture showed a high frequency of comorbid conditions; hypertension prevailed in some cohorts [8,9]. Both

${ }^{\star}$ Correspondence to: Shchudlo Michael, FSBI (Federal State Budget Institution) Russian Ilizarov Scientific Center "Restorative Traumatology and Orthopaedics", Clinical and Experimental Laboratory of Reconstructive-Plastic Microsurgery and Hand Surgery, Ul'ianova Street, 6. Kurgan, Russia, 640014, E-mail: nshchudlo@mail.ru

Key words: arterial hypertension, Dupuytren's contracture, palmar fascia, histomorphometry

Received: February 20, 2020; Accepted: February 26, 2020; Published: February 29, 2020 
Shchudlo N (2020) Clinical course of fascial fibromatosis, vascularization and tissue composition of Palmar Aponeurosis in patients with Dupuytren's Contracture and concomitant arterial hypertension

Dupuytren's contracture [7] and essential hypertension are characterized by similar changes in the small arteries that meet the criteria for accelerated vascular aging [10]. Features of the restructuring of blood vessels with a combination of these diseases and their possible influence on the clinical course of palmar fascial fibromatosis are unknown. Such information is necessary for the development of the theory of pathogenesis and methods of preventive and anti-relapse Dupuytren's contracture treatment.

\section{Aim}

The comparative analysis of fascial fibromatosis clinical course, vascularization and tissue composition of palmar aponeurosis in Dupuytren's contracture patients with normal arterial pressure and concomitant arterial hypertension.

\section{Materials and methods}

Case reports and surgical specimens material from 140 Dupuytren's contracture patients treated in FSBI (Federal State Budget Institution) Russian Ilizarov Scientific Center "Restorative Traumatology and Orthopaedics" in 2014-2018. Inclusion criteria - men aged 43-77 years (age range in which patients with both normal blood pressure and hypertension are presented), presence of contracture with clinically and histologically confirmed palmar fascial fibromatosis. Criteria of exclusion were multiple injuries in life history. The study was approved by the institution's ethics committee (protocol № 2 (57) of 19.03.2018) and were conducted in accordance with the ethical standards set out in the 1975 Helsinki Declaration, revised in 2008. According to case reports data patients were divided into two groups: "Dupuytren's contracture-normotension" (DCN) - $\mathrm{n}=61$ and "Dupuytren's contracture-hypertension" (DCH) $-\mathrm{n}=79$. In $\mathrm{DCH}$ group patients with 2-3 stages of hypertensive disease received antihypertensive therapy (calcium antagonists, $\beta$-blockers, angiotensin-converting enzyme inhibitors, blockers of receptors to angiotensin II in the form of monotherapy and various combinations including with thiazide diuretics). For comparative clinical characteristics of the groups the following criteria were used: age at the beginning of the palmar fascial fibromatosis (PFF), age at the time of surgery, body mass index, systolic arterial blood pressure (SABP), diastolic arterial blood pressure, per cent of patient with $\mathrm{SABP}>140$ at the time of the preoperative examination by the therapist, PFF duration (from the moment of the appearance of areas of skin compaction on the palm), stage of Dupuytren's contracture according to Tubiana's classification [11], number of fingers with impaired function. Surgical specimens of pathologically changed palmar aponeurosis were embedded in paraffin. Sections were cut at 5-7 $\mu \mathrm{m}$ on «Reichert» microtome (Austria) and stained with hematoxylineosin. For differentiated detection of smooth muscle cells, elastic and collagen fibers Masson's trichrome, van Gieson's and Weigert-van Gieson's methods were used. Part of material after aldehyde-osmium fixation were embedded in Araldite for semi thin $(0.5$ to $1.0 \mu \mathrm{m})$ slides. Sections were prepared on "Nova" ultra-tome LKB (Sweden) and stained with Toluidine Blue or Methylene Blue-Basic Fuchsin. Digital images of view fields were taken with photomicroscope "Opton-3" (Germany) connected with the "DiaMorph" hardware and software complex (Russia, Moscow). Histomorphometry was accomplishemed with PhotoFiltre and "VT-Master-Morphology" (VideoTest, Russia, St. Petersburg) programs. In images of transverse sections of the palmar aponeurosis arteries (magnification 500x) their external diameters were measured and formed samples of arteries with a diameter of 300 to 662 microns. These are the most significant for vascularization of palmar skin and distal parts of the hand branches of common digital arteries of the first order, perforating palmar aponeurosis. In samples of such arteries their lumen diameters, intima and media thickness were measured. Control - histologic slides of palmar aponeurosis fragments from three patients with acute open hand trauma. At a magnification of 200x, 30 view fields were digitized from each tissue sample. Using the electronic version of the test grid, the following percentage ratios were determined by the point-counting planimetry method: adipose tissue, loose, hyperplastic and dense connective tissues, blood vessels and sweat glands. In adipose tissue numerical and volumetric densities of blood vessels per cents of vessels with signs of lymphocytic-histiocytic infiltration and adventitial fibrosis were calculated.

For statistical processing of quantitative data, the Shapiro-Wilk test was used. In some samples the hypothesis about normality was rejected. Numerical data in tables are presented in the form of medians and quartiles (Me (Q1; Q3)). The hypothesis of differences was checked by nonparametric Wilcoxon, chi-square and exact Barnard tests. All statistical treatments were accomplished by software package Attestat Program (version 9.3.1, developed by I. P. Gaidyshev, Certificate of Rospatent official registration No. 2002611109).

\section{Results}

In group DCH compared with DCN patients were older by 5 years at the beginning of PFF and by 7 years at the time of surgery, respectively $(p<0,001)$. DCH group had a significantly greater body mass index $(p<0,01)$, systolic and diastolic blood pressure and per cent of patients with SBP $>140 \mathrm{~mm} \mathrm{Hg}$ at the time of the preoperative examination by the therapist $(\mathrm{p}<0,001)$. In DCH group PFF duration was insignificantly bigger $(p>0,05)$, stage of contracture - significantly $(p<0,05)$. Numbers of fingers with impaired function in studied groups were comparable (Table 1). In none of the groups did the stage of contracture correlate with the duration of the disease.

Histological examination of the surgical specimens established that in both groups of DC patients some palmar aponeurosis perforating arteries were little changed (Figure 1A). Others were characterized with lumen narrowing, hyperplastic media and presence of cells with contractile nuclei as part of the last (Figure 1B). In some arteries, neointimal hyperplasia was expressed with polymorphism of the cellular composition of the neointimal layer (Figure $1 \mathrm{C}$ and 1D). Special staining revealed the destruction of the elastic framework of the arteries, fibrosis of adventitia and neointimal layer (Figure 1D).

Among vessels of microcirculatory bed in both groups the arterioles with altered intimal and medial structures were noted, with fully or partialy obliterated lumens (Figure $2 \mathrm{~A}$ and $2 \mathrm{C}$ ). In some patients with controlled hypertension good safety of endothelial layer and microvessels lumens were marked (Figure $2 \mathrm{~B}$ and $2 \mathrm{D}$ ).

Morphometric analysis found that in both groups of DC patients compared with control the external and lumen diameters of arteries were significantly decreased and intimal thickness increased (Table 2). Such changes were significantly less dramatic in DCH group than in DCN group.

Stereologic analysis of palmar aponeurosis tissue composition revealed that in DCH group compared with DCN volumetric densities of adipose tissue and hyperplastic connective tissue were significantly smaller but the per cents of loose and dense connective tissues were bigger (Table 3). All parameters of vascularization in DCH group were smaller but per cents of vessels with signs of lymphocytic-histiocytic infiltration and adventitial fibrosis were significantly bigger. 
Shchudlo N (2020) Clinical course of fascial fibromatosis, vascularization and tissue composition of Palmar Aponeurosis in patients with Dupuytren's Contracture and concomitant arterial hypertension

Table 1. The main clinical patients' characteristics $(\mathrm{Me}(\mathrm{Q} 1 \div \mathrm{Q} 3))$

\begin{tabular}{|c|c|c|c|}
\hline Comparison criteria & $\begin{array}{c}\text { DCN } \\
(n=61)\end{array}$ & $\begin{array}{c}\text { DCH } \\
(n=79)\end{array}$ & $\mathbf{p}$ \\
\hline Age at the beginning of the palmar fascial fibromatosis (PFF) & $\begin{array}{c}49 \\
(45 \div 55)\end{array}$ & $\begin{array}{c}54 \\
(48 \div 61)\end{array}$ & $1,6 \times 10^{-5}$ \\
\hline Age at the time of surgery & $56(52 \div 61)$ & $63(57 \div 68)$ & $8,0 \times 10^{-5}$ \\
\hline Body mass index & $25(23 \div 28)$ & $27(25 \div 30)$ & $2,5 \times 10^{-3}$ \\
\hline Systolic arterial blood pressure (SABP) & $130(120 \div 130)$ & $140(140 \div 150)$ & $2,9 \times 10^{-12}$ \\
\hline Diastolic arterial blood pressure & $80(80 \div 80)$ & $90(80 \div 90)$ & $3,0 \times 10^{-6}$ \\
\hline $\begin{array}{l}\% \text { of patient with } \mathrm{SABP}>140 \text { at the time } \\
\text { of the preoperative examination by the therapist }\end{array}$ & 4,69 & 33,74 & $1,8 \times 10^{-5}$ \\
\hline PFF duration & $5(3 \div 10)$ & $7(3 \div 10)$ & 0,40 \\
\hline Stage of Dupuytren's contracture & $2,5(2 \div 3)$ & $3(2 \div 3)$ & 0,02 \\
\hline Number of fingers with impaired function & $2(1 \div 3)$ & $2(1 \div 3)$ & 0,22 \\
\hline
\end{tabular}

Note: DCN - normotensive patients with Dupuytren's contracture; DCH - patients with Dupuytren's contracture and arterial hypertension.

Table 2. Histomorphometric characteristics of perforant arteries $(\mathrm{Me}(\mathrm{Q} 1 \div \mathrm{Q} 3))$ of palmar aponeurosis

\begin{tabular}{|c|c|c|c|}
\hline Parameter/ group & External diameter & Luminal diameter & Intima thickness \\
\hline thickness
\end{tabular}

Note: ${ }^{1}$ - significant difference from control, ${ }^{2}$ - significant difference between normotensive and hypertensive patients with Dupuytren's contracture (DCN and DCH groups).

Table 3. Stereologic characteristics $(\mathrm{Me}(\mathrm{Q} 1 \div \mathrm{Q} 3))$ of tissue composition and vascularization of palmar aponeurosis

\begin{tabular}{|c|c|c|c|}
\hline Groups/parameters & $\begin{array}{c}\text { DCN } \\
(n=22)\end{array}$ & $\begin{array}{c}\text { DCH } \\
(n=28)\end{array}$ & $\mathbf{p}$ \\
\hline of fat tissue & $27,94(16,71 \div 31,86)$ & $24,45(20,68 \div 29,21)$ & $8,1 \times 10^{-12}$ \\
\hline$\%$ of loose connective tissue & $8,16(3,71 \div 10,79)$ & $12,81(6,01 \div 17,07)$ & $1,1 \times 10^{-16}$ \\
\hline$\%$ of hyperplastic connective tissue & $18,37(6,73 \div 24,86)$ & $14,15(3,09 \div 23,91)$ & $0,8 \times 10^{-16}$ \\
\hline$\%$ of dense connective tissue & $37,11(26,31 \div 44,44)$ & $42,47(32,42 \div 55,09)$ & $5,7 \times 10^{-15}$ \\
\hline$\%$ of vessels & $7,42(4,82 \div 9,18)$ & $4,93(2,51 \div 5,91)$ & $0,9 \times 10^{-16}$ \\
\hline$\%$ of sweat glands & $1,01(0,18 \div 1,06)$ & $1,17(0,12 \div 1,46)$ & 0,10 \\
\hline Numerical vessels density in the fat tissue & $5,25(3 \div 7)$ & $3,06(3 \div 3,5)$ & 0,048 \\
\hline Volumetric vessels density in the fat tissue & $2,03(0,89 \div 1,91)$ & $1,63(1,06 \div 2,09)$ & 0,34 \\
\hline$\%$ of vessels with signs of inflammatory infiltration & 10,86 & 17,29 & $4,0 \times 10^{-4}$ \\
\hline$\%$ of vessels with adventitial fibrosis & 7,67 & 14,28 & $3,9 \times 10^{-5}$ \\
\hline
\end{tabular}

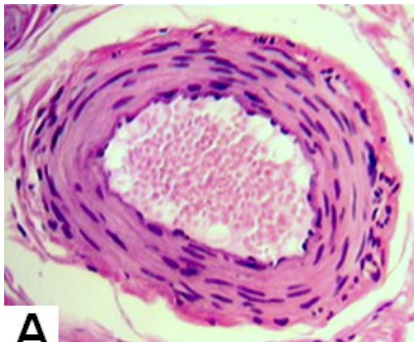

A
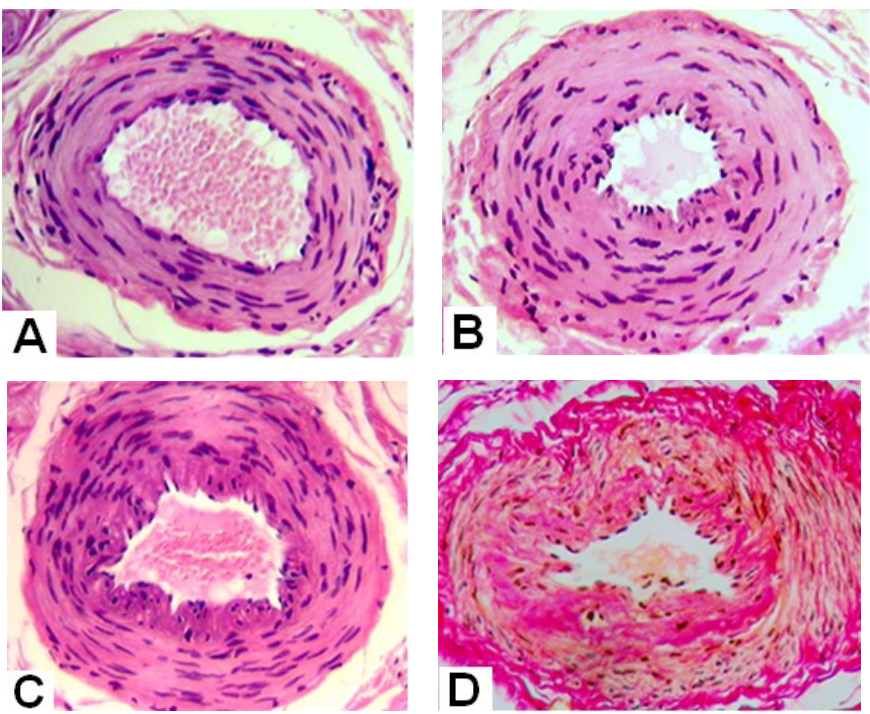

B

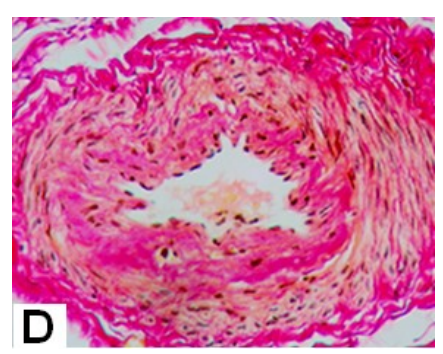

Figure 1. Transverse paraffin sections of palmar fascia arteries from patients with Dupuytren's contracture. Hematoxylin and eosin $(\mathbf{A}, \mathbf{B}, \mathbf{C})$ and Van Gieson (D) staining. Magnification $200 \mathrm{x}$
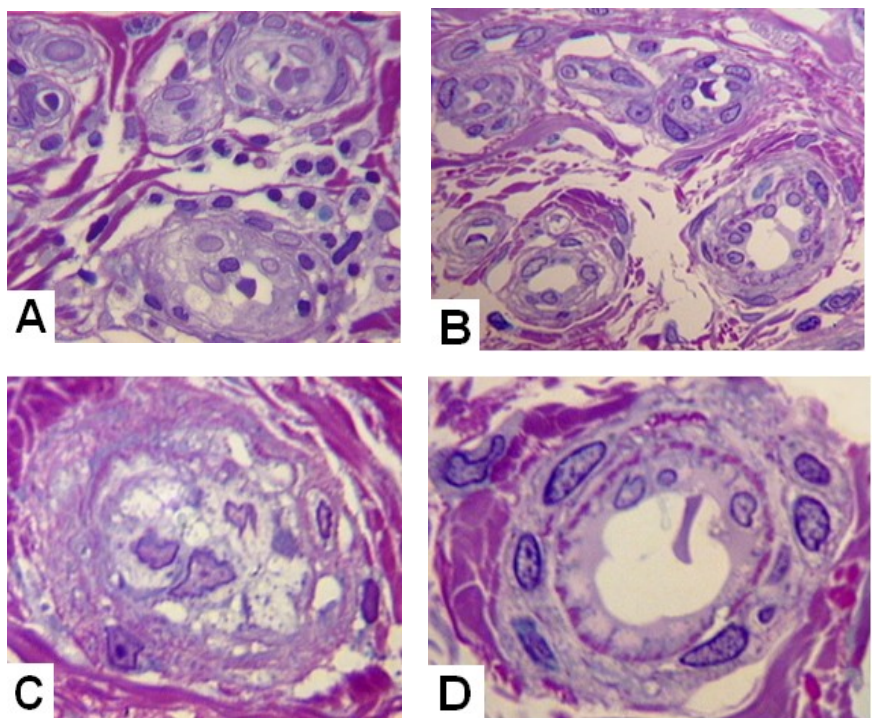

Figure 2. Transverse semithin sections of microvessels in palmar aponeurosis $(\mathbf{A}, \mathbf{C})$ from patient with Dupuytren's contracture and normotension, $(\mathbf{B}, \mathbf{D})$ - from patient with Dupuytren's contracture and concomitant arterial hypertension. Methylene Blue-Basic Fuchsin. Magnification 1250x, oil immersion.a 
Shchudlo N (2020) Clinical course of fascial fibromatosis, vascularization and tissue composition of Palmar Aponeurosis in patients with Dupuytren's Contracture and concomitant arterial hypertension

\section{Discussion}

The change in the caliber and lumen of the vessels (remodeling) is a key event in the pathogenesis of many diseases $[10,12,13]$. It was established in experimental and clinical research that during arterial hypertension the active myogenic vasoconstriction leads to eutrophic internal remodeling of small arteries, which consists in the rearrangement of smooth muscle cells and extracellular matrix around the narrowed lumen without changes in the number and mass of cells [14]. Vessels role in Dupuytren's contracture pathogenesis was estimated inconsistently. According to some authors, the high rates of latent vasospasm in digital arteries are a constitutional phenomenon unrelated to fibromatosis [15]. Others believe that it is the narrowing of the vessels of the palmar aponeurosis that triggers hypoxia and activation of xanthine oxidase, leading to transformations of fibroblasts [16]. Our study revealed histomorphometric features of palmar aponeurosis arteries remodeling in Dupuytren's contracture men with normal blood pressure or with arterial hypertension. In both groups compared with control (fragments of palmar aponeurosis from patients with acute open hand trauma without fascial fibromatosis) arteries caliber and lumen were reduced due to adventitious fibrosis, shrinkage of the muscular layer and the formation of a neointimal layer. Previously by other authors, it was found that such changes (constrictive arterial remodeling) are carried out under conditions of enhanced proliferation of adventitious fibroblasts and myofibroblasts migrating into the internal layers of the arterial wall [17]. Myofibroblasts possessing the properties of both fibroblasts and contractile smooth muscle cells also plays a major role in the development of fascial fibromatosis $[18,19]$. It should be assumed that concomitant hypertension exacerbates the narrowing of the arteries in palmar aponeurosis of patients with Dupuytren's contracture. However, our study of such patients revealed less pronounced constrictive arterial remodeling than in patients with Dupuytren's contracture and normotension. The most likely explanation is the vasodilating effect of antihypertensive drugs. The main limitation of the study -it was impossible to obtain exact information about antihypertensive therapy in retrospective analysis. But about $66 \%$ of patients of DCH group had a systolic blood pressure of no more than 140 at the moment of the preoperative examination by a therapist, testifying to the effective pharmacological control of hypertension. Vasodilators used to lower blood pressure slowed down not only constrictive remodeling of the vessels of the palmar aponeurosis, but also the development of the initial signs of palmar fascial fibromatosis. This is evidenced by older patients in DCH group at the beginning of the disease and at the time of surgery. But in that group with concomitant hypertension more advanced contracture and more advanced histologic stage of fibromatosis were marked [20].

Thus, in our study, the dual role of pharmacologically controlled concomitant arterial hypertension in the course of the palmar fascial fibromatosis was revealed. It begins at a later age, but in many patients leads to more severe contracture and a more advanced histological stage of fibromatosis at the time of surgery. Therefore, when prescribing antihypertensive therapy to patients with palmar fascial fibromatosis, information available in the scientific literature on the possible pro- and antifibrotic effects of antihypertensive drugs of different groups [21,22] should be taken into account.

\section{Conclusions}

1. Patients with Dupuytren's contracture and concomitant pharmacologically controlled arterial hypertension are characterized by a later onset of the palmar fascial fibromatosis than at normal arterial pressure. This is presumably associated with the less severe constrictive remodeling of the perforating arteries of the palmar aponeurosis identified in this study, which was probably delayed by antihypertensive vasodilating therapy.

2. In cases with concomitant arterial hypertension clinical and pathological data indicate accelerated development of fascial fibromatosis, which is caused by the rarefication of the microvasculature.

\section{Financial disclosure}

supported by the RF Ministry of Health within governmentmandated program for FSBI Russian Ilizarov Scientific Center "Restorative Traumatology and Orthopaedics" (RISC "RTO") for Scientific Research in 2018-2020 Yr./Yrs. №AAAA-A18-118011190119-5 from 11/01/2018.

\section{Conflict of interests}

The authors declare that they have no conflict of interest.

\section{References}

1. Vishnevskiy VO (2014) Dupuytrenā contracture of hand and its medical and social review. Zaporozhye medical journal 1: 912. [In Russian].

2. 2. Mandel DR, de Marco PJ. Overview of the pathogenesis, diagnosis and treatment of Dupuytren's disease. Int J Clin Rheumatol 9: 217-225.

3. Loos B, Puschkin V, Horch RE (2007) 50 years experience with Dupuytren's contracture in the Erlangen University Hospital - A retrospective analysis of 2919 operated hands from 1956 to 2006. BMC Musculoskelet Disord 8: 60.

4. van Nuffel M, Degreef I (2014) Pharmacotherapy, steroid injection, fat graft, PIP external fixators and other techniques. In: Handchirurgie weltweit e.V (Ed) Living Textbook of Hand Surgery.

5. Murrell GA, Francis MJ, Howlett CR (1989) Dupuytren's contracture. Fine structure in relation to aetiology. J Bone Joint Surg Br 71: 367-373. [Crossref]

6. Mayerl C, Del Frari B, Parson W, Boeck G (2016) Characterisation of the inflammatory response in Dupuytren's disease. J Plast Surg Hand Surg 50: 171-179. [Crossref]

7. Shchudlo N, Varsegova T, Stupina T, Dolganova T, Shchudlo M, et al. (2018) Arteria remodelling in palmar fascia and peculiarities of hand microhemodynamics in patients with Dupuytren's contracture. Rheumatol Orthop Med 3: 1-7.

8. Mansur HG, Oliveira ER, Gonēalves CB (2017) Epidemiological analysis of patients with Dupuytren's disease. Rev Bras Ortop 53: 10-14. [Crossref]

9. Shchudlo N, Varsegova T, Stupina T, Dolganova T, Shchudlo M, et al. (2018) Assessment of palmar subcutaneous tissue vascularization in patients with Dupuytren's contracture. World J Orthop 9: 130-137.

10. Early Vascular Aging (EVA) (2015) New Directions in Cardiovascular Protection. Peter Nilsson, Michael Olsen, Stephane Laurent (Eds) Academic Press p. 376

11. Tubiana R (1999) Dupuytren's disease of the radial side of the hand. Hand Clin 15 149-159. [Crossref]

12. Morreale M, MulĆØ G, Ferrante A, D'ignoto F, Cottone S (2016) Early Vascular Aging in Normotensive Patients With Systemic Lupus Erythematosus: Comparison With Young Patients Having Hypertension. Angiology 67: 676-682.

13. Nicolosi PA, Tombetti E, Maugeri N, Rovere-Querini P, Brunelli S, et al. (2016) Vascular Remodelling and Mesenchymal Transition in Systemic Sclerosis. Stem Cells Int 2016: 4636859.

14. Prewitt RL, Rice DC, Dobrian AD (2002) Adaptation of resistance arteries to increases in pressure. Microcirculation 9: 295-304.

15. 15. Bauer M, Hussl H, HĆ pfel-Kreiner I, Polaczek R, SchlĆ ๆgel R. Blood flow in the finger in Dupuytren's contracture. Plethysmographic, histological and neurological findings. Chirurgia plastica 5: 243-248.

16. Murrell GA (1991) The role of the fibroblast in Dupuytren's contracture. Hand Clin 7: 669-680. [Crossref]

17. Geary RL, Nikkari ST, Wagner WD, Williams JK, Adams MR, et al. (1998) Wound healing: a paradigm for lumen narrowing after arterial reconstruction. J Vasc Surg 27: 96-106. 
Shchudlo N (2020) Clinical course of fascial fibromatosis, vascularization and tissue composition of Palmar Aponeurosis in patients with Dupuytren's Contracture and concomitant arterial hypertension

18. Tomasek JJ, Vaughan MB, Haaksma CJ (1999) Cellular structure and biology of Dupuytren's disease. Hand Clin 15: 21-34. [Crossref]

19. Feihl F, Liaudet L, Waeber B, Levy BI (2006) Hypertension: a disease of the microcirculation? Hypertension 48: 1012-1017.

20. Schiffrin EL (2012) Vascular remodeling in hypertension: mechanisms and treatment. Hypertension 59: 367-374.
21. Agrawal P, Gautam A, Bansal S, Doneria J, Pursnani N (2018) Metoprolol Induced

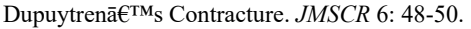

22. Knobloch K, Redeker J, Vogt PM (2009) Antifibrotic medication using a combination of N-acetyl-L-cystein (NAC) and ACE inhibitors can prevent the recurrence of Dupuytren's disease. Med Hypotheses 73: 659-661.

Copyright: $\mathbb{C} 2020$ Shchudlo N. This is an open-access article distributed under the terms of the Creative Commons Attribution License, which permits unrestricted use, distribution, and reproduction in any medium, provided the original author and source are credited. 